\title{
ON THE RENAL EXCRETION OF XYLOSE ${ }^{1}$
}

\author{
By R. DOMINGUEZ AND ELIZABETH POMERENE
}

(From the Laboratories of Saint Luke's Hospital, Cleveland)

(Received for publication May 4, 1934)

The excretion of xylose has been investigated as a further application of the method of analysis which we have already applied to the excretion of ingested creatinine (1). The method consists in analyzing separately the time change in the rate of excretion by the kidney and in the plasma concentration, after the initial disturbance produced by the rapid absorption of creatinine and its distribution in the body fluids has subsided. It was found that, in the case of creatinine, both the rate of excretion and the plasma concentration diminish exponentially in the course of time, the two functions being asymptotic to their pre-ingestion values, and furthermore, that the coefficients of the time in both exponentials are equal, on an average. Under these conditions, it was proved that the elimination of the time between pairs of simultaneous equations yields a linear relation between the rate of output and the instantaneous concentration in the plasma. It was also proved that the method provides the means of determining the errors of observation. After the constants had been calculated and the errors of observation taken into consideration, it was shown that practically all the variation in the ratio of the rate of output to the plasma concentration (called glomerular filtration rate (2) or creatinine clearance (3)) could be accounted for.

The growing literature on xylose has prompted us to study the excretion of this substance by the same method. The same interpolating functions have been found to hold as in the case of creatinine, namely, simple exponential functions of the time, asymptotic to the pre-ingestion blank levels. The only difference in the behavior of the two substances, outside the values of the constants, is that the exponential phase of decrease in plasma concentration $(x)$ and rate of output $(y)$ begins somewhat later after the ingestion of xylose than after creatinine. The two exponential curves will be referred to as the $x$ curve and the $y$ curve respectively.

Six experiments were performed in two healthy human beings, who will be referred to as Subjects $E$ and $D$. Subject $E$ is the same one whose data on creatinine have been reported (1).

\footnotetext{
1 Aided by a grant from the Florence $H$. Kridler Fund.

This work was presented before the American Physiological Society at its forty-sixth annual meeting, March 29th, 1934, New York City.
} 
The material will be presented briefly as follows: 1 , chemical methods ; 2 , endogenous blanks; 3 , experiments; 4 , constants of the functions; 5 , analysis of errors; and 6, the excretion constant of xylose.

\section{CHEMICAL METHODS}

The details of the methods of collection and preservation of plasma and urine are given in a previous publication (1). Duplicate and sometimes triplicate analyses were made on the plasma and urine samples, all of which had been renumbered by an independent worker so as to obviate bias. The Folin and Wu (4) method with d-xylose (Pfanstiehl) standards of appropriate strength was used for the determination of the reducing substances of urine and the nonfermentable reducing substances of plasma. For color matching a Klett Biocolorimeter with north light was used. The intensity of illumination was greatly cut down when the strength of dilute solutions was determined (5). It was found under the conditions observed that the reducing power of plasma and urine varied insignificantly during a four day period, the maximum time for a complete series of analyses for an experiment.

For the removal of the yeast-fermentable reducing substances of plasma the method of Blanco (6) was found to yield the most consistent results and, within the limits of experimental error, complete recovery of added xylose. In every series of plasma determinations, yeast blanks were run as a check on the washing of the cells. Consistent results were obtained throughout with tungstic acid extracts of plasma and yeast standing at room temperature for from 15 minutes up to 2 hours before centrifuging and decanting the supernatant fluid. The total amount of reducing substances in plasma and yeast, before the ingestion of xylose, will be regarded as the blank, and its mean value from a number of determinations will be called the endogenous blank of plasma concentration.

The reducing substances of urine gave less consistent values when yeast was used, although added xylose was completely recovered. Consequently the determination of reducing substances was made without yeast on urines diluted with distilled water so that $1 \mathrm{cc}$. of the final dilution, the minimum amount pipetted, contained no more than $0.4 \mathrm{mgm}$. of reducing substances.

Endogenous blanks. The total nonfermentable reducing substances of oxalated plasma plus yeast were, for Subject E, mean of 7 determinations, $6.46 \mathrm{mgm}$. per $100 \mathrm{cc}$., standard error of mean 0.9989; for Subject D, mean of 7 determinations, $6.87 \mathrm{mgm}$. per $100 \mathrm{cc}$., standard error of mean 0.7144 .

The output of reducing substances in urine were, for Subject $E$, mean of 19 determinations, $0.9037 \mathrm{mgm}$. per minute, standard error of mean 0.0373 ; for Subject D, mean of 9 determinations, $1.0595 \mathrm{mgm}$. per minute, standard error of mean 0.0474 . There is no significant difference between 
the two subjects as far as their endogenous blanks for plasma concentration are concerned, but the difference between their endogenous outputs of reducing substances, small as it is, can be shown to be statistically significant.

\section{EXPERIMENTS}

The protocols are given in Table I. In all the experiments diarrhea of varying degree developed, more marked with the larger amounts ingested.

TABLE I

Experimental data on xylose excretion

\begin{tabular}{|c|c|c|c|c|c|}
\hline \multirow[t]{2}{*}{$\begin{array}{l}\text { Experimental } \\
\text { details }\end{array}$} & $\begin{array}{l}\text { Time of urine } \\
\text { collection }\end{array}$ & $\begin{array}{c}\text { Urine } \\
\text { volume }\end{array}$ & $\begin{array}{l}\text { Urine } \\
\text { xylose }\end{array}$ & $\begin{array}{l}\text { Time of } \\
\text { blood } \\
\text { collec- } \\
\text { tion }\end{array}$ & $\begin{array}{l}\text { Plasma } \\
\text { xylose }\end{array}$ \\
\hline & & $c c$. & $\begin{array}{c}\underset{\text { mgm. }}{\text { per } 100} \\
\text { cc. }\end{array}$ & & $\begin{array}{c}\underset{\text { per } 100}{\text { mgm. }} \\
\text { cc. }\end{array}$ \\
\hline \multirow{10}{*}{$\begin{array}{l}\text { Subject E, female, white; body weight } \\
45 \mathrm{kgm} . \text {; standing height } 159 \mathrm{~cm} \text {. } \\
\text { Experiment } E-1, \text { November } 15,1933 \text {. } \\
50 \text { grams xylose, } 9.28 \text { a.m.; break- } \\
\text { fast, } 7.20 \text { a.m. Routine labora- } \\
\text { tory work during this and subse- } \\
\text { quent experiments. d-xylose, } \\
\text { Pfanstiehl, by mouth in all experi- } \\
\text { ments. }\end{array}$} & $8.24-9.27$ & 163 & 37.6 & 9.23 & 4.5 \\
\hline & $9.27-10.09$ & 131 & 205 & 10.01 & 16.1 \\
\hline & $10.09-10.39$ & 28 & 2823 & 10.31 & 44.8 \\
\hline & $10.39-11.38$ & 53 & 3545 & 11.33 & 62.2 \\
\hline & $11.38-12.38$ & 69 & 3940 & 12.25 & 69.5 \\
\hline & $12.38-1.44$ & 57 & 4380 & 1.30 & 49.6 \\
\hline & $1.44-2.43$ & 38 & 3895 & 2.32 & 31.6 \\
\hline & $2.43-3.51$ & 36 & 3293 & 3.34 & 25.4 \\
\hline & $3.51-4.48$ & 25 & 2898 & 4.35 & 21.3 \\
\hline & $4.48-5.44$ & 19 & 2380 & 5.37 & 13.9 \\
\hline \multirow{12}{*}{$\begin{array}{l}\text { Experiment } E-2, \text { December } 4,1933 . \\
25 \text { grams xylose, } 8.45 \text { a.m.; break- } \\
\text { fast, } 7.15 \text { a.m. }\end{array}$} & $7.30-8.10$ & 219 & 22 & 8.36 & 4.9 \\
\hline & $8.10-8.43$ & 179 & 20 & 9.45 & 34.5 \\
\hline & $8.43-9.50$ & 122 & 747 & 10.43 & 35.1 \\
\hline & $9.50-10.49$ & 437 & 360 & 11.32 & 29.7 \\
\hline & $10.49-11.38$ & 380 & 275 & 12.29 & 18.3 \\
\hline & $11.38-12.29$ & 264 & 325 & 1.25 & 13.3 \\
\hline & $12.29-1.35$ & 320 & 149 & 2.33 & 12.1 \\
\hline & $1.35-2.21$ & 476 & 54.8 & 3.46 & 7.6 \\
\hline & $2.21-2.53$ & 331 & 40.8 & 4.35 & 8.1 \\
\hline & $2.53-3.35$ & 363 & 37.8 & & \\
\hline & $3.35-4.09$ & 257 & 31.1 & & \\
\hline & $4.09-4.44$ & 235 & 30.2 & & \\
\hline \multirow{9}{*}{$\begin{array}{l}\text { Experiment } E-3, \text { January } 10,1934 . \\
25 \text { grams xylose, } 9.08 \text { a.m.; break- } \\
\text { fast, } 7 \text { a.m }\end{array}$} & $8.08-9.07$ & 99 & 61 & & \\
\hline & $9.07-10.14$ & 47.5 & 1768 & & \\
\hline & $10.14-11.10$ & 55.5 & 3258 & & \\
\hline & $11.10-12.15$ & 106 & 1824 & & \\
\hline & $12.15-1.17$ & 124 & 1010 & & \\
\hline & $1.17-2.17$ & 52 & 1295 & & \\
\hline & $2.17-3.17$ & 149 & 287 & & \\
\hline & $3.17-4.14$ & 213 & 133 & & \\
\hline & $4.14-5.14$ & 32 & 566.5 & & \\
\hline
\end{tabular}


TABLE I (continued)

\begin{tabular}{|c|c|c|c|c|c|}
\hline $\begin{array}{l}\text { Experimental } \\
\text { details }\end{array}$ & $\begin{array}{l}\text { Time of urine } \\
\text { collection }\end{array}$ & $\begin{array}{c}\text { Urine } \\
\text { volume }\end{array}$ & $\begin{array}{c}\text { Urine } \\
\text { xylose }\end{array}$ & $\begin{array}{l}\text { Time of } \\
\text { blood } \\
\text { collec- } \\
\text { tion }\end{array}$ & $\begin{array}{l}\text { Plasma } \\
\text { xylose }\end{array}$ \\
\hline \multirow{10}{*}{$\begin{array}{l}\text { Subject } D \text {, male, white; body weight } \\
70 \text { kgm.; standing height } 179 \mathrm{~cm} \text {. } \\
\text { Experiment } D-1, \text { November } 23,1933 \text {. } \\
50 \text { grams xylose, } 8.59 \text { a.m.; break- } \\
\text { fast, } 8.15 \text { a.m. }\end{array}$} & & $c c$. & $\begin{array}{c}\underset{\text { mgm. }}{\text { per } 100} \\
\text { cc. }\end{array}$ & & $\begin{array}{c}\text { mgm. } \\
\text { per } 100 \\
\text { cc. }\end{array}$ \\
\hline & $7.50-8.55$ & 46.5 & 158 & 8.53 & 5.3 \\
\hline & $8.55-9.59$ & 98.5 & 312 & 9.58 & 84.4 \\
\hline & $9.59-10.56$ & 97.5 & 3605 & 10.55 & 91.8 \\
\hline & $10.56-11.55$ & 100 & 3235 & 11.52 & 69.4 \\
\hline & $11.55-1.14$ & 82.5 & 2963 & 1.10 & 38.1 \\
\hline & $1.14-2.06$ & 36.5 & 2410 & 2.03 & 20.8 \\
\hline & $2.06-3.13$ & 42.5 & 1736 & 3.05 & 14.9 \\
\hline & $3.13-4.09$ & 28.5 & 1353 & 4.07 & 13.0 \\
\hline & $4.09-5.15$ & 27 & 1163 & 5.07 & 10.8 \\
\hline \multirow{9}{*}{$\begin{array}{l}\text { Experiment } D-2, \text { December } 13,1933 \text {. } \\
25 \text { grams xylose, } 9.32 \text { a.m.; break- } \\
\text { fast at } 8.30 \text { a.m. Part of the urine } \\
\text { of the fourth period was lost before } \\
\text { the volume was measured. The } \\
\text { volume estimated from the cre- } \\
\text { atinine concentration is } 158.4 \mathrm{cc} \text {. }\end{array}$} & $8.28-9.30$ & 71.5 & 110 & 9.34 & 5.6 \\
\hline & $9.30-10.41$ & 144.5 & $14 i 5$ & 10.40 & 65.2 \\
\hline & $10.41-11.43$ & 219 & 904 & 11.42 & 43.9 \\
\hline & $11.43-12.36$ & & 914 & 12.35 & 37.0 \\
\hline & $12.36-1.46$ & 106 & 1064 & 1.45 & 19.0 \\
\hline & $1.46-2.39$ & 85.5 & 511 & 2.37 & 16.3 \\
\hline & $2.39-3.39$ & 49 & 662 & 3.37 & 7.7 \\
\hline & $3.39-4.39$ & 50 & 420 & 4.38 & 9.4 \\
\hline & $4.39-5.39$ & 107.5 & 144 & 5.37 & 7.0 \\
\hline \multirow{8}{*}{$\begin{array}{l}\text { Experiment } D-3, \text { January } 10,1934 . \\
25 \text { grams xylose, } 10 \text { a.m.; break- } \\
\text { fast, } 8.30 \text { a.m. Five minutes } \\
\text { were lost between the third and } \\
\text { fourth periods due to defecation. }\end{array}$} & $7.55-9.58$ & 174 & 84.3 & & \\
\hline & $9.58-11.14$ & 118.5 & 1639 & & \\
\hline & $11.14-12.20$ & 143.5 & 1763 & & \\
\hline & $12.25-1.48$ & 152 & 1592 & & \\
\hline & $1.48-2.45$ & 44 & 1783 & & \\
\hline & $2.45-3.47$ & 55 & 846 & & \\
\hline & $3.47-4.39$ & 44 & 572 & & \\
\hline & $4.39-6.15$ & 66 & 458.5 & & \\
\hline
\end{tabular}

Constants of the fitted functions. The fitted functions were:

$$
\begin{aligned}
& y=y_{e}+a e^{-\alpha t}, \\
& x=x_{e}+b e^{-\beta t},
\end{aligned}
$$

where $y$ is the rate of excretion of xylose, in mgm. per minute, $x$ the concentration of xylose in mgm. per $100 \mathrm{cc}$. of plasma, $y_{e}$ and $x_{e}$ the endogenous blanks already given, $e$ the base of natural logarithms, $t$ the time in hours, and $a, b, a$, and $\beta$ constants to be determined from the data. Inasmuch as the method of calculation is the same as that used for creatinine (1), all details will be omitted. However, there is one point that calls for a brief comment. In one of the experiments it was found impossible to fit the points satisfactorily by least squares applied to the weighted logarithms. It was necessary to go back to the classical method of least squares after reducing the fitting function to a linear form by Taylor's theorem. 
The constants $a$ and $\beta$ are given in Table II. The ratio $(\alpha / \beta)$ from pairs of simultaneous equations is $0.9975,0.9621,0.9022$, and 1.1948, mean 1.014 , so that $a$ and $\beta$ are practically equal, which establishes the linearity of the relation between the plasma concentration and the rate of output.

\section{TABLE II}

Exponentials fitted to excretion rate $(y)$ and plasma concentration $(x)$ of xylose

$$
y=y_{e}+a e^{-\alpha t}, \quad x=x_{e}+b e^{-\beta t}
$$

\begin{tabular}{|c|c|c|c|c|c|c|}
\hline Experiment & $\begin{array}{l}\text { Amount } \\
\text { ingested }\end{array}$ & $\alpha . \log e$ & $\begin{array}{c}\text { Number of } \\
\text { observations }\end{array}$ & $\beta . \log e$ & $\begin{array}{l}\text { Number of } \\
\text { observations }\end{array}$ & $\vec{V} \dagger$ \\
\hline $\begin{array}{l}\text { D-1 } \\
\text { D-2 } \\
\text { D-3 }\end{array}$ & $\begin{array}{c}\text { grams } \\
50 \\
25 \\
25\end{array}$ & $\begin{array}{l}0.2297 \\
0.2754 \\
0.2868\end{array}$ & $\begin{array}{l}6 \\
5 \\
5\end{array}$ & $\begin{array}{l}0.2546 \\
0.2305\end{array}$ & $\begin{array}{l}6 \\
7\end{array}$ & $\begin{array}{l}0.835 \\
1.314 \\
1.005\end{array}$ \\
\hline $\begin{array}{l}\text { E-1 } \\
\text { E-2 } \\
\text { E-3 }\end{array}$ & $\begin{array}{l}50 \\
25 \\
25\end{array}$ & $\begin{array}{l}0.1656^{*} \\
0.2340 \\
0.2412\end{array}$ & $\begin{array}{l}5 \\
8 \\
5\end{array}$ & $\begin{array}{l}0.1660^{*} \\
0.2432\end{array}$ & $\begin{array}{l}6 \\
6\end{array}$ & $\begin{array}{l}0.661 \\
7.673 \\
1.924\end{array}$ \\
\hline
\end{tabular}

Mean $(\alpha . \log e)=0.254$, mean $(\beta . \log e)=0.243$.

* Excluded from mean.

$+\bar{V}=$ mean of urine rates for whole experiment, in cc. per minute.

It should be remarked that if the exponentials are made asymptotic to zero (as done by Fishberg ( 7 ) as far as the plasma concentration is concerned), the values of $a$ and $\beta$ would be definitely different from each other, and the resulting relation between $y$ and $x$ would not be linear.

Analysis of errors. From the result of Table II, that a mean value can be obtained for $a(=$ mean $\beta$ ), new values can be computed for $a$ and $b$, and from these, theoretical values for $y$ and $x$, let us say, $Y$ and $X$ respectively. The differences $(y-Y)$ and $(x-X)$ are the deviations of $y$ and $x$ from their respective mean curve, and are independent of each other. Experiment E-1 cannot be included in this analysis because of the low value of $a$ (and $\beta$ ). This low value, probably due to delayed absorption, will be considered in a future publication.

Errors of $x$. Nineteen residuals were obtained with constants and distribution as follows:

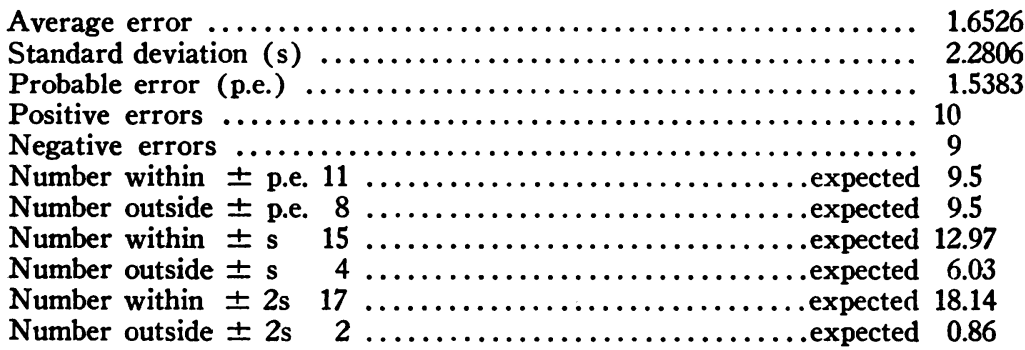


The mean is -0.3684 with standard error $=0.5234$, so that the mean is not significantly different from zero.

Errors of $y$. Twenty-nine residuals were available with constants and distribution as follows:

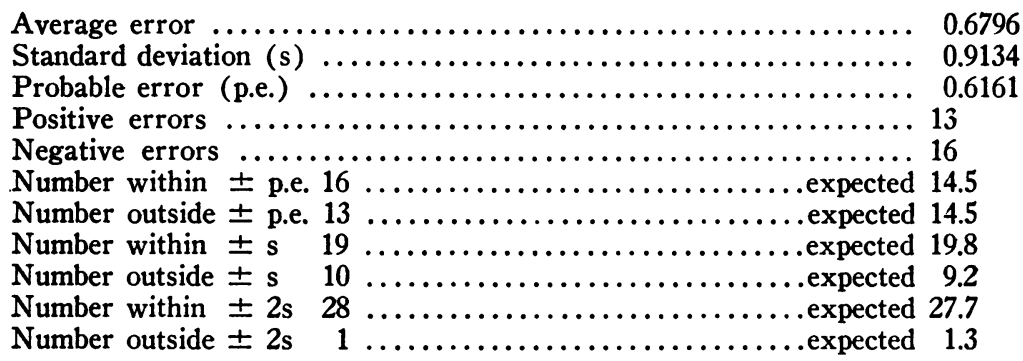

The mean is -0.0546 with standard error 0.1696 . The mean is, therefore, not significantly different from zero.

A comparison with a similar analysis of the creatinine residuals (1) shows that the errors of the data on xylose are larger, especially the errors of the plasma concentration, as was to be expected from the nature of the chemical methods employed. On the other hand, the distribution of the errors of xylose output is considerably closer to the normal distribution of errors, indicating that perhaps idiosyncrasies in one subject have been neutralized by mixing the data of two different subjects.

The excretion constant of $x y l o s e$. If in the process of fitting, the time of ingestion has been reckoned as the zero-time, Equations 1 and 2 are automatically synchronized. The elimination of the time between these two equations yields the relation,

$$
\left(y-y_{e}\right)=A\left(x-x_{e}\right),
$$

where $A=(a / b)$ is the excretion constant of xylose.

The value of $A$ from four simultaneous experiments is as follows: D-1, 0.690; D-2, 0.815; E-1, 0.777; E-2, 0.817; mean $A, 0.77$. In Experiment $\mathrm{E}-1$ the calculation was made directly from the fitted equations, but in Experiments E-2, D-1, and D-2 the constant $A$ was computed after recalculation of $a$ and $b$ (see Equations 1 and 2 ) using the mean value of $(a \cdot \log e)=0.25$, or $a=0.5756$, instead of the values of $a$ or $\beta$ given in Table II.

It should be realized that, although the errors of the time can be looked upon as negligible as compared with the errors of $y$ or $x$, it is by no means certain that the synchronization of two pairs of curves insures the simultaneity of the events. Theoretically at least, the $y$ curve lags behind the $x$ curve, because of the time elapsing between the formation of urine and its excretion. Besides, the $y$ points have been computed as loaded ordinates in the middle of the interval of urine collection, but since the fall in 


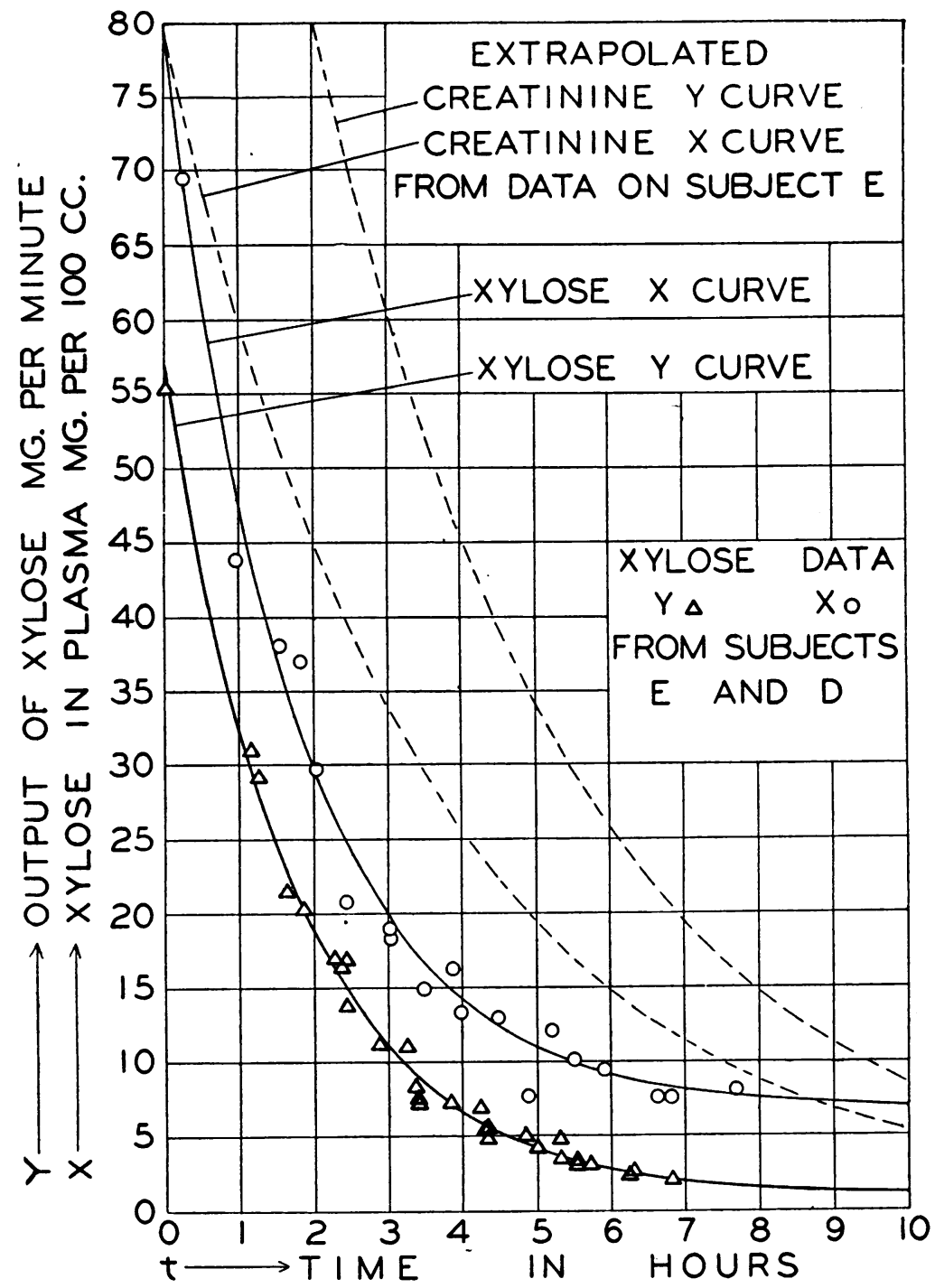

Fig. 1. Time Change in Plasma Concentration and Rate of Output of Xylose in Man

The equation of the plasma curve ( $x$ curve) is $x=6.87+72.65 e^{-0.576 t}$, that of the rate of output ( $y$ curve) is $y=1.06+55.94 e^{-0.576 t}$. The observations of plasma concentration are represented by circles, those of rate of output by triangles. The corresponding curves of the data on creatinine (broken lines) have been drawn by extrapolating from the published equations (1) so that the plasma concentration of both substances begins at the same level. The asymptotes have not been drawn in order to avoid confusion. The larger scattering of the circles about the $x$-curve should be noted. The time given has no reference to the time of ingestion of the substance. 
the rate of excretion is exponential, the mean rate of an interval does not lie theoretically on the midpoint of the interval. These two sources of error should be added to the ones already discussed (1) as influencing the final value of the excretion $A$.

Figure 1 shows the mean curves for the time change of the plasma concentration and the rate of output of xylose. The two corresponding curves for creatinine (extrapolated from the published equations (1)) have been drawn to enable comparison. In order to avoid confusion, the asymptotes have not been drawn. The larger scattering of the observations (represented in the figure by circles) about the curve of plasma concentration is evident. The difference in the endogenous output of reducing substances between the two subjects would have been imperceptible in the figure, and consequently no attempt was made to distinguish the points in the original drawing.

\section{DISCUSSION}

The method of analyzing the changes in plasma concentration and rate of output of a substance with respect to time, although incomplete, in that the initial phase following ingestion has not been considered, supplies a number of useful quantitative relations to be discussed in future publications. Here only the linearity of the resultant equation will be considered.

Equation 3 can be written

$$
y=p+A x,
$$

where

$$
p=y_{e}-A x_{e} .
$$

Substituting in Equation 4 the numerical values of the constants we obtain

$$
\begin{aligned}
& \text { Subject E } y=-4.0705+0.77 x, \\
& \text { Subject D } y=-4.2304+0.77 x .
\end{aligned}
$$

The same situation is found here, therefore, that was found in the analysis of the creatinine data (1), namely, that the straight line representing the relation between the rate of output of xylose and the plasma concentration does not go through the origin of the coordinate system. Consequently the ratio $(y / x)$, called "glomerular clearance" by Shannon, Jolliffe and Smith (8) is affected not only by the errors in $y$ and $x$ (and the larger errors are in the denominator), but also by a systematic error arising from the fact that $p$ (Equation 4) is not zero. The ratio, from Equation 4, is

$$
(y / x)=A+(p / x)
$$

and is evidently a function of the plasma concentration. This systematic 
error becomes larger the smaller the plasma concentration, but it is not due to errors in the latter.

In order to give a clear idea of the extent of the variation in the ratio from all the sources mentioned, it will be enough to calculate the ratios (clearances) for the data of this report. For Subject $E$ the mean of the ratios is 0.51 , with a range of 0.25 to 0.72 ; for Subject $D$, mean 0.52, range 0.32 to 0.70 . The mean of the ratios is, therefore, far below the excretion constant calculated from the same data, and the variation of the ratio is such as to discourage any use of it. Of course, having assigned to this ratio a specific meaning, that of measuring the glomerular filtrate, the authors above mentioned regard this variation as quite in keeping with accepted views on the variability of the glomerular filtrate (see "Discussion" in (8)).

If, instead, a straight line is fitted to the very points the ratios of whose coordinates show such variation, the two equations result:

$$
\begin{aligned}
& y=-3.258+0.729 x, \\
& y=-3.427+0.736 x,
\end{aligned}
$$

according as $x$ or $y$, respectively, is assumed free from error. It is obvious then that the fitting of a straight line to data roughly interpolated during their exponential decrease would succeed, where the ratios had failed, in giving a close approximation to the constant calculated by the general method (Equations 6 and 7 ).

From Equation 8 it is also clear that the larger the plasma concentration the closer will the ratio approximate to the value of the excretion constant, the degree of approximation depending also on $p$. Hence, papers in which the ratios only are published are of no assistance in the calculation of the excretion constant. For this reason we are unable to analyze the data of Jolliffe and Chasis (9) on man. If the plasma concentration of their subjects was around $50 \mathrm{mgm}$. per $100 \mathrm{cc}$., as mentioned by Jolliffe and Chasis, their ratios, calculated from Equation 6 or 7, would be about 10 per cent below the value of the excretion constant. This will exaggerate the discrepancy between the excretion constant of our report (0.77) and the mean ratio of theirs $(0.97$ after correcting for the difference in units). Further comparison of their data with ours is rendered difficult by the circumstance that the points used for our calculations were taken from a different part of the curve. Our data were taken, as underscored above, during the exponential decrease of both curves, theirs apparently in the region of the maximum. It is not known whether identical relations hold for the whole curve, but even assuming such identity, the linear interpolation used to synchronize the data is unreliable in the region of the maximum. Whatever the case might be, we shall simply note that the excretion constant of Subjects $E$ and D lies in the lower range of the ratios 
recorded by Jolliffe and Chasis. The reader's attention is called to the fact that, in order to make this comparison, the published ratios of Jolliffe and Chasis should be multiplied by the surface area of the respective subject and corrected for units.

As a further illustration of the fallacy of the ratio, the recent report of Shannon (10) on the dogfish will be analyzed. From his summary and conclusions we shall quote the following:

"At plasma levels below $7 \mathrm{mgm}$. per cent the creatinine clearance appears to reach a maximum, varying in our experiments from 4.2 to 7.2, and averaging 5.8 times the xylose or sucrose clearance. As the plasma level is raised to higher values the creatinine clearance falls, approaching the xylose or sucrose clearance asymptotically."

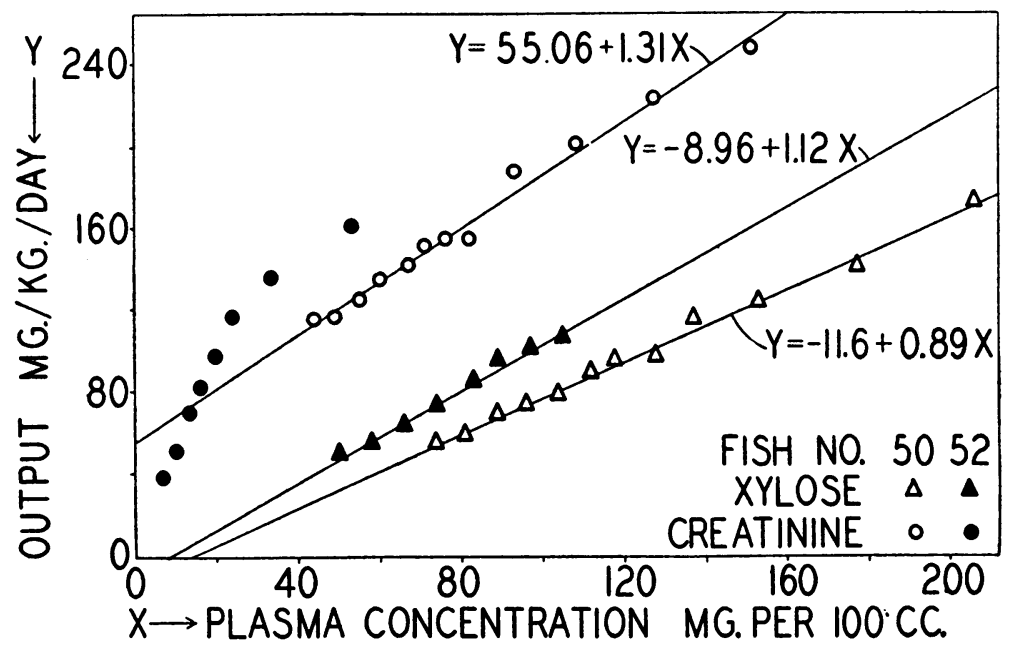

Fig. 2. Straight Lines Fitted to the Data of Shannon (10) on the Excretion of Xylose and Creatinine in the Dogfish

The observations on xylose are represented by triangles, those on creatinine by circles. The data from one fish (Number 50 ) are indicated by open symbols, those on the other (Number 52) by closed symbols. The points fall along straight lines except those of creatinine in Fish 52. Note the large intercepts of the three lines drawn, especially that corresponding to the creatinine data on Fish 50, giving rise to a systematic variation in the "glomerular clearance," ratio $(y / x)$.

Since only two protocols were published, from two different animals, and no data were given in regard to the endogenous blanks, the general method of computation cannot be applied. But we can calculate the outputs from the published ratios (Table II (10)) and pair them with the (interpolated) plasma concentrations as given. By plotting on rectangular coordinates the following figure is obtained (Fig. 2). It is at once seen that the points of xylose fall along straight lines cutting the $x$-axis to the 
right of the origin, as in the human case for xylose and creatinine, whereas the creatinine points show a remarkable behavior. Those of Fish 50 fall on a straight line intercepting the $y$-axis at $y=55.0 \mathrm{mgm}$. per kilo per day, but those of Fish 52 do not follow a straight line at all. Whether this is the normal behavior of the renal excretion of creatinine at low plasma levels (say below $25 \mathrm{mgm}$. per $100 \mathrm{cc}$.) in the dogfish, is not clear from the data, but whether it is so or not it is quite clear that the ratio $(y / x)$ cannot be used. Leaving the creatinine data of Fish 52 out of consideration, the other three lines can be calculated as shown in the figure (see Fig. 2). Calculating the ratios for these equations, we get

$$
\begin{array}{ll}
\text { Fish 50, creatinine, } & (y / x)=1.31+(55.06 / x), \\
\text { Fish 50, xylose, } & (y / x)=0.89-(11.60 / x) .
\end{array}
$$

Consequently, the creatinine ratio will increase as the plasma creatinine decreases, and the xylose ratio will decrease as the plasma xylose decreases. Since the quantity $p$ is larger for creatinine than for xylose, and the plasma concentrations are in general larger for xylose than for creatinine, it follows that the effect of a changing plasma concentration will be more pronounced with creatinine than with xylose. All these conclusions can be verified by examining Table II of Shannon's report (10) or by direct calculation from Equations 11 and 12.

Furthermore, if the ratio of the ratios is considered, as has usually been done, and one is careful not to use small values of plasma xylose which make the double ratio grow to infinity and then become negative, it will be seen, from Equations 11 and 12, that, for increasingly large values of plasma creatinine and plasma xylose, the double ratio will behave roughly as a decreasing function of the plasma creatinine alone. This behavior deceived Shannon as to the character (he calls it logarithmic) of the function. The function is algebraic, and, for large constant values of plasma xylose, its asymptote is not 1 , as stated in the last sentence of the above quotation, but 1.47, equal to the ratio of the two excretion constants.

The theoretical considerations of Shannon, based, as they are, on a fictitious variation, are, of course, meaningless, but his experimental data should be carefully reexamined, because the numerous though erratic observations in the dog (11) suggests a close numerical value for the ratio of the two excretion constants in these two widely separated animal species (1.37 for the dog, 1.47 for the dogfish).

It would take an unnecessary amount of space to analyze the data on the dog, for which reason we shall limit ourselves to a group of data on one $\operatorname{dog}(11)$. Twenty-three observations are available, 12 from a long experiment, and 11 from three short experiments. By treating the latter 
as if supplied by one experiment, and calculating the straight lines, we get

Dog 36, creatinine (long experiment) $y=0.616+0.659 x$, (13)

Dog 36, creatinine (short experiment) $y=0.161+0.569 x$, (14)

Dog 36, xylose (long experiment) $y=0.377+0.524 x,(15)$

Dog 36, xylose (short experiment) $y=3.395+0.384 x$. (16)

Although the creatinine data were obtained by two different methods of analysis, and moreover the plasma concentration of creatinine was in general much less than that of xylose, the data on xylose show by far the larger scattering and the coefficients of $x$ (excretion constants) the greater discrepancy, confirming not only the relative inaccuracy of the determination of xylose concentration, but also our general conclusion that the variation in the ratio is due to errors of analysis, chemical or otherwise. Here too, we see that the constant term of the equations (Equations 13 to 16) will introduce a systematic variation in the ratio, but this term being small in magnitude (with the exception of that in Equation 16), the effect will be appreciable only at low plasma levels, and consequently will be easily detected in the creatinine ratios, but not at all evident in the xylose ratios. If we separate the ratios as given in the table (Table I (11)) according as the plasma concentration of creatinine is larger than $25 \mathrm{mgm}$. per $100 \mathrm{cc}$. or less, we get, for the mean of the ratios in the first group (10 observations), 79.3, and for the mean of the ratios in the second group (13 observations), 99.9. The authors of the paper quoted, naturally, attach no significance to this variation, because in their experience the ratios of the dog vary " between 40 and 150 per sq. m.," but, in view of the fact that the variation in the ratio includes all the variations that can occur in the kind of experiments usually made, it will be immediately seen that the excretion constant cannot exhibit such disconcerting variation, even if uncorrected for the surface area or the weight of the animal.

Pending a better determination of the constants we can say that the excretion constant of creatinine in the dog is 1.26 or 1.48 times that of xylose, according as we take Equations 13 and 15, or Equations 14 and 16. All the double ratios (Table I (11), column 10) fall within these limits.

In man, as far as the observations on our Subject $E$ are concerned, the excretion constant of creatinine is 2.36 times as large as that of xylose, and is, therefore, in the upper range of the double ratios calculated by Jolliffe and Chasis (9). Since in calculating the ratio of two simultaneous " clearances" the urine flow cancels out, the double ratio is nothing more than the ratio of two concentration indices. For the double ratio to approximate, within its wide variation limits, to the ratio of two excretion constants it is necessary and sufficient that the excretion constants be independent of urine flow. That the excretion of xylose is independent of 
urine flow is shown by examining the large fluctuation of urine flow in our experiments (Table I) or the mean diureses in Table II, especially those of Subject E, and noticing that such fluctuations have no effect either on the regularity of the curves or on the distribution of the errors. That the excretion of creatinine is also independent of urine flow has been shown previously (1).

In regard to the physiological significance of the excretion constant, even if considered as the corrected value of a "clearance," we can say, supplementing what was said a propos of creatinine, that the exponential law is one of the few fundamental relations between two magnitudes and is shared by a multitude of physical and chemical phenomena. It would be unjustified, therefore, from the exponential decrease of plasma concentration and rate of output, or from its consequence, the linear relationship between these two quantities, to draw any conclusions about the intricate mechanism whereby a substance is removed from the plasma.

A large number of inert substances can be imagined to be excreted by the kidney in a manner similar to that of creatinine or xylose, which may give a corresponding series of constants. And, if these constants could be arranged according to their magnitude in the same order as that of some physical property of the substances, their diffusion constants, for instance, the information so derived might lead to a better understanding of the inner working of the kidney.

Finally, it should be pointed out, that, from the superficial agreement between the variability of the "clearance" and the variability of the glomerular filtration rate, it cannot be argued that the relative stability of the excretion constant is incompatible with its representing the filtration rate, because the instability of every one of the elements of an assemblage does not preclude the stability of the whole, witness body temperature, diastolic blood pressure, etc.

\section{SUMMARY AND CONCLUSIONS}

The excretion of xylose has been investigated by a method previously used, which determines the time change of plasma concentration and rate of output of the substance during their phase of exponential decrease. The method establishes the linearity of the relation between these two quantities, supplies the necessary constants, and besides gives explicit information on the errors of observation. The excretion constant of xylose, calculated from data on two human subjects, is 0.77 , when the plasma concentration $(x)$ is measured in $\mathrm{mgm}$. per $100 \mathrm{cc}$. and the rate of output $(y)$ in mgm. per minute. This constant is independent of moderate exercise and moderate changes in diuresis.

The behavior of the two quantities ( $y$ and $x$ ) with respect to time is illustrated graphically and the corresponding curves of creatinine, previously obtained, are also included to enable comparison. 
Other published data on man are either too incomplete to be analyzed by this method, or are given only in the form of a ratio $(y / x)$.

It is shown that the ratio of the two quantities $(y / x)$ is disturbed not only by accidental errors of observation (and the larger errors are in the denominator), but also systematically by the fact that the straight line satisfying the relation between $y$ and $x$ does not go through the origin of the coordinate system. This systematic variation in the ratio is found to be relatively unimportant in the case of the dog, but quite serious in the published data on the dogfish. The danger of interpreting variations in the ratio in terms of renal physiology is made obvious.

\section{BIBLIOGRAPHY}

1. Dominguez, R., and Pomerene, E., Studies of the renal excretion of creatinine. I. On the functional relation between the rate of output and the concentration in the plasma. J. Biol. Chem., 1934, 104, 449.

2. Rehberg, P. B., Studies on kidney function. I. The rate of filtration and reabsorption in the human kidney. Biochem. J., 1926, $20,447$.

3. Cope, C. L., The excretion of creatinine by the human kidney in health and in nephritis. Quart. J. Med., 1931, 24, 567.

4. Folin, O., and Wu, H., A system of blood analysis. Supplement I. A simplified and improved method for determination of sugar. J. Biol. Chem., 1920, 41, 367.

5. Folin, O., and Svedberg, A., The sugar in urine and in blood. J. Biol. Chem., 1926, 70, 405.

6. Blanco, J. G., Sugar metabolism. Lactose, galactose and xylose. J. Biol. Chem., 1928, 79, 667.

7. Fishberg, E. H., The rate of disappearance of foreign sugar from the blood stream. J. Biol. Chem., 1930, 86, 665.

8. Shannon, J. A., Jolliffe, N., and Smith, H. W., The excretion of urine in the dog. IV. The effect of maintenance diet, feeding, etc., upon the quantity of glomerular filtrate. Am. J. Physiol., 1932, 101, 625.

9. Jolliffe, N., and Chasis, H., The filtration and secretion of exogenous creatinine in man. Am. J. Physiol., 1933, 104, 677.

10. Shannon, J. A., Absorption and excretion of water and salts by the elasmobranch fishes. IV. The secretion of exogenous creatinine by the dogfish, Squalus acanthias. J. Cell. and Comp. Physiol., 1934, 4, 211.

11. Shannon, J. A., Jolliffe, N., and Smith, H. W., The excretion of urine in the dog. VI. The filtration and secretion of exogenous creatinine. Am. J. Physiol., 1932, 102, 534. 